\title{
Functional analysis of the 5' flanking domain of the LOXL4 gene in head and neck squamous cell carcinoma cells
}

\author{
TIBOR GÖRÖGH ${ }^{1}$, CLAUDIA HOLTMEIER ${ }^{2}$, JAN BERND WEISE ${ }^{1}$, MARKUS HOFFMANN ${ }^{1}$, \\ PETRA AMBROSCH${ }^{1}$, MARTIN LAUDIEN ${ }^{1}$ and KATALIN CSISZAR ${ }^{3}$ \\ ${ }^{1}$ Division of Experimental Oncology, Department of Otorhinolaryngology, Head and Neck Surgery and \\ ${ }^{2}$ Department of Dermatology, University of Kiel, D-24105, Germany; ${ }^{3}$ Laboratory of Matrix Pathobiology Pacific \\ Biomedical Research Center, University of Hawaii, HI, USA
}

Received May 16, 2008; Accepted August 12, 2008

DOI: 10.3892/ijo_00000098

\begin{abstract}
Lysyl oxidases are a family of five copperdependent amine oxidases including LOX, LOXL, LOXL2, LOXL3 and LOXL4. LOX and LOXL are essential for the assembly and maintenance of extracellular matrixes. LOXL2, LOXL3 and LOXL4, secreted and active enzymes, were also noted in association with diverse tumor types. We have recently reported overexpression of the LOXL4 mRNA and protein and a close relation of LOXL4 with the pathogenesis of head and neck squamous cell carcinomas (HNSCC). In this study, we analyzed the organization of the LOXL4 gene and addressed the regulatory mechanisms responsible for the overexpression. We demonstrated de novo transcription of the LOXL4 gene in HNSCC, but not in normal squamous epithelial cells. Analysis of the consecutive promoter region spanning positions -960 to -1 identified binding sites for several transcription factors. Promoter constructs containing selected specific promoter regions and consensus binding sites exhibited significantly increased reporter gene activity in HNSCC cells, but not in normal epithelial cells in transient coexpression experiments. The activity profiles of some of these constructs were similar in both cell types indicating that elements of the basic transcriptional regulatory mechanisms remained intact in HNSCC cells. DNA-binding experiments demonstrated that nuclear extracts from HNSCC cells have increased binding activity to the TATA $(-25)$ and the SP1 (-181) sites compared to normal epithelial cells, suggesting that these transcription factors are involved in the upregulation of LOXL4 gene expression in HNSCC.
\end{abstract}

Correspondence to: Dr Tibor Görögh, Department of Otorhinolaryngology, Head and Neck Surgery, University of Kiel, ArnoldHeller-Str. 14, D-24105 Kiel, Germany

E-mail: gorogh@hno.uni-kiel.de

Key words: head and neck carcinoma, LOXL4 gene structure, transcription factor

\section{Introduction}

Lysyl oxidases are a family of five copper-dependent amine oxidases including LOX, LOXL, LOXL2, LOXL3 and LOXL4. LOX and LOXL are essential for the assembly and maintenance of the structure of extracellular matrix (ECM). The active LOX was recently reported to play essential roles in invasive breast carcinoma and LOXL2, 3 and 4, similarly active enzymes, were also noted in association with diverse tumor types. The LOXL4 gene encodes an enzyme of $84,5 \mathrm{kDa}$ that is synthesized with a 24-residue amino acid $\mathrm{N}$-terminal signal peptide and secreted into the ECM (1). The LOXL4 transcript is about $4 \mathrm{~kb}$ and is expressed in various tissue types including the pancreas and testes (2). The C-terminal region of LOXL4 contains domains that are homologous to highly conserved C-terminal domains present in all the LOX proteins and are known to be associated with their catalytic activity. The N-terminal region of LOXL4 has four scavenger receptor domains that are also conserved within the LOXL2 and LOXL3 isoforms.

LOX (EC 1.4.3.13) catalyzes the oxidation of lysine residues to semialdehydes in its substrates, fibrillar collagens and elastin, that then serve as precursors for the covalent cross-linkages stabilizing these fibers in the ECM (3). The secreted LOX is a $50 \mathrm{kDa}$ proenzyme that is processed into a biological active $30 \mathrm{kDa}$ form by BMP1 (4). LOX has been noted in different tissues in both extra- and intracellular locations, having related but distinct functions $(2,4-8)$. Importantly, we have reported an essential role for the active LOX in inducing invasive behaviour in breast tumour epithelial cells though activation of FAK/Scr signalling (9) and an association with invasive breast tumours (10). These novel functions were further confirmed in a mouse model for invasive breast cancer (11).

Our recent analyses of molecular alterations in HNSCC cells identified another member of the LOX family, LOXL4, as one of the mRNAs with the highest HNSCC specific expression in comparison to normal epithelial cells (12). Subsequently, we found an association of LOXL4 with the pathogenesis of head and neck squamous cell carcinomas and reported that in HNSCC cells overexpression of LOXL4 was associated with an increased dosage of the LOXL4 gene 
due to an isochromosome of the long arm of chromosome 10 (13).

To address additional mechanisms that contributed to LOXL4 overexpression in HNSCC, in this study we have analyzed the genomic and mRNA structure of LOXL4 and its de novo expression in HNSCC cells along with control normal epithelial cells. We have evaluated the activity of a series of LOXL4 promoter-reporter gene constructs that contained selected transcriptional factor binding sites. Furthermore, we tested the differential nuclear extract binding activities of these sites in tumour vs. normal squamous epithelial cells.

\section{Materials and methods}

Cell culture. UT-SCC-19A laryngeal and HTB-117 pharyngeal carcinoma cells were grown in minimum essential medium (Biochrome, Berlin, Germany) at $37^{\circ} \mathrm{C}$ in a $5 \% \mathrm{CO}_{2}$ humidified atmosphere. For control experiments normal epithelial cells were derived from histologically confirmed non-dysplastic mucosa of resected laryngeal carcinomas. All tissues for cell culture were retrieved following informed consent approved by the local ethics committee (AZ:D413/03). The normal epithelial cells were grown in serum-free medium (Bio Whittaker, Apen, Germany), and for confirmation of their squamous origin cytokeratin immunostaining profile was routinely performed using antibodies directed against cytokeratins 5, 6, 10 and 13. Keratinization was observed by positive staining with anti-cytokeratin 5 and 6 .

Isolation of total RNA. For rapid and reproducible preparation of total RNA, the TRIzol reagent (Invitrogen, Karlsruhe, Germany) was used in accordance with the manufacturer's instruction. After measurement of the RNA concentration, samples of total RNA were subjected to subsequent firststrand cDNA synthesis. Quantitative analysis of the mRNA content was done by RT-PCR using glyceraldehyde 3-phosphate dehydrogenase GAPDH as a control.

First-strand cDNA synthesis. Total RNA $(1 \mu \mathrm{g})$ was heat denatured $\left(70^{\circ} \mathrm{C}, 10 \mathrm{~min}\right)$, chilled on ice and subjected to oligo(dT)-primed reverse transcription. The procedure was conducted with 300 units of MMLV reverse transcriptase (Invitrogen) in the presence of $5 \mu 1$ of $2 \mu \mathrm{M}$ dNTP and 40 units of RNAse inhibitor (Life Technologies, Inc.) in a total volume of $20 \mu \mathrm{l}$ (adjusted with DEPC warter) for $60 \mathrm{~min}$ at $35^{\circ} \mathrm{C}$. The resulting double-stranded RNA:cDNA heteroduplex was heat denatured at $95^{\circ} \mathrm{C}$ for $5 \mathrm{~min}$ to provide cDNA as template for amplification.

Amplification of the 5' cDNA region. 5' RACE was performed to amplify the upstream region of the LOXL4 mRNA, that we identified by differential display-PCR as selectively expressed in SCC cell lines (12), using the Smart Race cDNA Amplification Kit and the Advantage 2 PCR Enzyme System (Clontech, Heidelberg, Germany). The resulting clones were sequenced using the dye terminator sequencing method (Applied Biosystems). Sequences were aligned with known database sequences using the BLAST engine at the National Center for Biotechnology Information (NCBI). Verification of the sequence was performed on mRNA that was isolated from UTSCC-19A cells using the quick prep RNA purification method (Qiagen, Hilden, Germany).

Analysis of the exon-intron organization of the LOXL4 gene. After amplification of the 5 ' end of the differentially expressed mRNA, all available databases were screened to identify sequences that shared homologies with the LOXL4 cDNA. A human genomic sequence, accession number AL 139241, was identified and analyzed. Lack of overlap between the cDNA and genomic sequences was strictly confined to introns. To verify the positions and lengths of the introns high stringency PCR was carried out using oligonucleotides specific for LOXL4 exon-intron boundary sequences and genomic DNA isolated from normal squamous epithelial cells. The PCR products were purified, sequenced and the sequences aligned with the human genomic sequences AL 139241.

Nuclear run-on assay. Nuclei of UT-SCC-19A and normal epithelial cells were isolated and run-on experiments were performed as described elsewhere (14). After lysis of $2 \times 10^{7}$ cells in 1-ml ice-cold nuclear extraction buffer consisting of $3.6 \mathrm{mM} \mathrm{KCl}, 6 \mathrm{mM} \mathrm{CaCl} 2,1.2 \mathrm{mM} \mathrm{MgSO}_{4}, 6 \mathrm{mM}$ sodium citrate, $6 \mathrm{mM}$ sodium pyruvate, $6 \mathrm{mM}$ sucrose $12.5 \mathrm{mM}$ Tris, $50 \mathrm{mM}$ rifampicine, and $0.5 \%(\mathrm{v} / \mathrm{v})$ Nonidet-P40 nuclei were isolated by centrifugation at $4{ }^{\circ} \mathrm{C} 800 \mathrm{rpm}$ for $5 \mathrm{~min}$ and subjected to run-on transcription. Nuclei suspensions (50 $\mu \mathrm{l})$ were incubated for $90 \mathrm{~min}$ at room temperature in a total of $100 \mu 1$ reaction mixture containing $100 \mu \mathrm{Ci}\left[\alpha^{32} \mathrm{P}\right] \mathrm{UTP}$ (Amersham, Braunschweig, Germany), $1 \mu 1$ of $0.1 \mathrm{M}$ dithiothreitol (DTT), $1 \mu \mathrm{l}$ of $100 \mathrm{mM}$ each of ATP, CTP and GTP (Roche Molecular Biochemicals, Mannheim, Germany). Newly synthesized RNA was extracted using a commercial kit (TRIzol reagent, Invitrogen). Finally the RNA was dissolved in $200 \mu \mathrm{l}$ DEPC-treated water including $100 \mathrm{U}$ of RNaseinhibitor (RNase-Out, Invitrogen). Equal amounts $(5 \mu \mathrm{g})$ of purified LOXL4 -and G3PDH-cDNA were separated in agarose gel, vacuum-transferred onto Hybond-N membrane (Amersham) using the TDNA vacuum-blotter (Appligene, Heidelberg, Germany) and subjected to prehybridization at $42^{\circ} \mathrm{C}$ for $2 \mathrm{~h}$. Hybridization was performed at $42^{\circ} \mathrm{C}$ for $24 \mathrm{~h}$ in $30 \%$ STARKS containing $30 \%$ formamide (v/v), $0.02 \%$ BSA (w/v), $0.02 \%$ ficoll (w/v), $0.02 \%$ polyvinylpyrrolidone (w/v), $1 \%$ SDS in SSC (150 mM NaCl, $15 \mathrm{mM}$ sodium citrate) and the labeled RNA as probe. Membranes were then washed in $2 \mathrm{X} \mathrm{SSC}$ for $2 \times 20 \mathrm{~min}$ at $37^{\circ} \mathrm{C}$ and exposed to X-ray film for 4 days at $-80^{\circ} \mathrm{C}$ using an intensifier screen. Subsequently, signals were detected in a phosphorimager (Storm860; Molecular Dynamics, Heidelberg, Germany).

LOXL4 gene-regulatory constructs. The reference sequence representing a part of the human chromosome 10 that overlaps the LOXL4 genomic sequence is that of GenBank accession number AL139241. The 5' region from position +384 to -2554 was subdivided into 5 overlapping fragments. We have designed fragment-specific primers (FSP's) and carried out PCR amplification using genomic DNA from both UTSCC-19 cells and cells with normal epithelial phenotype. The FSPs used were: i) sense 5'-CAGATGAGGAGAC TGAGACTCAG-3', anti-sense 5'-GCAGCAGCAGGAACA 
GAAAGAGG-3'; ii) sense 5'-GTGCTAGGAACATCAGAA GGTGG-3', anti-sense 5'-CTGAGTCTCAGTCTCCTCATC TG-3'; iii) sense 5'-GTTGCAGTGAGCTGAGATTGCAG-3', anti-sense 5'-CCACCTTCTGATGTTCCTAGCAC-3'; iv) sense 5'-GGCAGGTCTCATCAGAACGAGC-3' anti-sense 5'-CTGCAATCTCAGCTCACTGCAAC-3'; v) sense 5'-GG AGAGCTGCAGAGGTGAGAATG-3', anti-sense 5'-GC TCGTTCTGATGAGACCTGCC-3'. After PCR the amplified products were inserted into the HindIII/BglII opened pCR2.1-TOPO cloning vector (Invitrogen) and sequenced on an automated sequencer (ABI Prism 310, Applied Biosystems, Weiterstadt, Germany).

Electrophoretic mobility shift assay (EMSA). UTSCC-19A and normal epithelial cells were washed in PBS, centrifuged at $800 \mathrm{rpm}$ and the pellets were resuspended in a buffer containing $30 \mathrm{mM}$ succrose, $0.5 \mathrm{ml}$ of $1 \mathrm{M}$ Tris $(\mathrm{pH} 8.0)$, $150 \mu 1$ of $1 \mathrm{M} \mathrm{CaCl}_{2}, 100 \mu \mathrm{l}$ of $1 \mathrm{M} \mathrm{MgOAc}, 10 \mu \mathrm{l}$ of $0.5 \mathrm{mM}$ PMSF, $1 \mu \mathrm{M}$ DTT, $0.5 \%$ (v/v) Nonidet-NP40 (Fluka Chemie, Buchs, Switzerland). After centrifugation at $500 \mathrm{rpm}$ for $5 \mathrm{~min}$, the pellets were washed in the same buffer without NonidetNP40 centrifuged again and resuspended in $100 \mu 1$ low salt buffer containing $1 \mathrm{mM}$ HEPES, $0.075 \mathrm{mM} \mathrm{MgCl}, 1 \mathrm{mM}$ $\mathrm{KCl}, 0.01 \mathrm{mM}$ EDTA (pH 8.0), 32\% glycerol, $0.5 \mathrm{mM}$ DTT and $0.5 \mathrm{mM}$ PMSF. Subsequently, nuclear extracts were treated with $40 \mathrm{mM}$ protease inhibitor (Roche Molecular Biochemicals) and protein concentration was measured as described (15). For the EMSA nuclear extracts (each of $1 \mu \mathrm{g}$ ) were incubated in a reaction mixture $(10 \mu 1)$ consisting of $0.5 \mu \mathrm{g}$ poly(dI-dC), $2 \mu 1$ binding buffer (50 mM Tris/pH 8.0, $750 \mathrm{mM} \mathrm{KCl,} 2.5 \mathrm{mM}$ EDTA, 0.5\% Triton-X 100 (v/v), 62\% glycerol (v/v), $1 \mathrm{mM}$ DTT and $\left[\gamma^{32} \mathrm{P}\right] \mathrm{dATP}-$ labeled oligonuleotides. The sequences of oligonucleotides used in this assay were: Reporter (REP)1: 5'-AGGTGTATAAGAG CTGCTGTTTATTGTGTACTCACCAATGCTAGG-3'; REP2: 5'-TAGATAGTCTTGACCTGAGGGCACCCTGGG GGCATCCCCGTGACTCAAATGGTGGACCACCTCG TCTGCC-3'; REP3: 5'-CATTTGATTAGGGTGCTAGGAA CATCAGAAGGTGGTGGCAGTGTCC CTGGGG-3'; REP4: 5'-CAGCCTTCCATGTGCCTTCTAGGTGAAGG CGGCACTTCA-3'; REP5: 5'-AGCCAATGAGTGGTGGA GTCCTTGATGCCCATCTAATAGCCCTTTCTGCTC-3'; REP6: 5'-TCGAAAAAAAAAAAAAAAAGAAAGAAA GAAAGAAAAAAGAAAGAAA-3'; REP7: -5'TGAGATTG CAGCACTGCAGTCCAGCCTGGGCGACAGAGAGAGA CTCTGTCT-3'. Each protein-DNA complex was analyzed using 5\% non-denaturing polyacrylamide gel at $10^{\circ} \mathrm{C}$ for $1 \mathrm{~h}$. After electrophoresis the gel was dried at $70^{\circ} \mathrm{C}$ for $1 \mathrm{~h}$ and exposed to X-ray film with an intensifying screen at $-80^{\circ} \mathrm{C}$.

Promoter constructs and cell transfections. For transient transfection experiments the REP fragments were extracted from the HindIII/BgIII opened pCR2.1-TOPO cloning vector (Invitrogen) and amplified by PCR using oligonucleotides containing flanking ends complementary to the enzyme restriction sites. After amplification and purification, the PCR products were cloned into the pGL3-enhancer-plasmid vector (Promega) and the reporter gene constructs were subjected to analyze Dual-Luciferase-Activity (DLA) using the Reporter-Assay-Kit (Promega). Final quantification of the Firefly- and the Renilla-Luciferase was performed by means of the Lumat LB-9507 luminometer (Berthold Technologies, Bad Wildbad, Germany) in a measurement interval of $10 \mathrm{sec}$.

Statistical analysis. Fisher's exact test was used to assess significant differences in LOXL4 promoter activity between HNSCC cells and controls with normal epithelial cell phenotype. Data were considered statistically significant at $\mathrm{p}$-value $\leq 0.05$.

\section{Results}

Organization of the LOXL4 gene at chromosome 10q24. Alignment of the LOXL4 cDNA sequences with database sequences identified a human genomic entry with accession number AL 139241. This genomic sequence of $30 \mathrm{~kb}$ derived from clone RP11-34A14 of chromosome 10, encompassed the entire LOXL4 gene including the 5' and 3'UTR sequences. Using this genomic sequence in comparison with the LOXL4 gene specific sequence that we previously identified (12), we determined the positions of the introns and all coding exons (Table I). The gene covers about $14.095 \mathrm{~kb}$ of the genomic sequence with an open reading frame of $2.278 \mathrm{~kb}$ and a $5^{\prime}$ UTR of 384 bases (Fig. 1A). Subsequently, we implemented a series of PCR experiments using genomic DNA derived from normal squamous epithelial cells and upstream and downstream primers specific for LOXL4 exon-intron boundary sequences. Using the sizes of the amplified fragments, we have confirmed the size of all 14 exons with lengths between $39 \mathrm{bp}$ and $278 \mathrm{bp}$ (Fig. 1B). The transcriptional start site was at position +384 upstream of the translation initiation codon (ATG) (Fig. 1C). In the 3' UTR region of the gene transcript a $1230 \mathrm{bp}$ untranslated trailer sequence was found downstream of the first stop codon TGA at position 14.480. Computer analysis of the 3 ' non-coding region of the reference LOXL4-cDNA, revealed a consensus polyadenylation signal located $30 \mathrm{bp}$ upstream of the poly-A tail. A second poly-adenylation signal was also identified 292 bp upstream of the first, suggesting that there may be an additional functional poly-A signal.

Differential expression of LOXL4 in HNSCC cells. Nuclear run-on transcription assay was used to assess whether the differential expression of the LOXL4-mRNA is the consequence of higher transcription rate of the LOXL4 gene in HNSCC cells or de novo transcription of the LOXL4 gene. From results presented in Fig. 2 it was evident that during the time of the transcription assay an active LOXL4 gene transcript was produced by polymerase reaction in HNSCC cells in contrast to normal epithelial cells which did not produce radiolabeled LOXL4-mRNA. Control experiments using a GAPDH gene-specific probe verified the reliability of the run-on transcription assay as indicated by the similar quantity of the mRNA for this house-keeping gene in both cell types.

Sequence and motif analysis of the promoter region. For functional tests of the 5' UTR of the LOXL4 gene, a total of seven consecutive and partially overlapping regions were 
Table I. Exon-intron organization of the LOXL4 gene.

\begin{tabular}{lccrr}
\hline Exon (no.) & Exon position & Exon size (bp) & Intron position & Intron size (bp) \\
\hline 1 & $385-662$ & 278 & $663-1191$ & 529 \\
2 & $1192-1370$ & 179 & $1371-2277$ & 907 \\
3 & $2278-2483$ & 206 & $2484-3975$ & 1492 \\
4 & $3976-4014$ & 39 & $4015-4176$ & 162 \\
5 & $4177-4396$ & 220 & $4397-5240$ & 844 \\
6 & $5241-5424$ & 184 & $5425-5600$ & 176 \\
7 & $5601-5757$ & 157 & $5758-6459$ & 702 \\
8 & $6460-6625$ & 166 & $6626-7665$ & 1040 \\
9 & $7666-7830$ & 165 & $7831-9600$ & 1770 \\
10 & $9601-9852$ & 252 & $9853-10936$ & 1084 \\
11 & $10937-11052$ & 116 & $11053-11702$ & 650 \\
12 & $11703-11840$ & 138 & $11841-12229$ & 389 \\
14 & $12230-12340$ & 111 & $12341-14413$ & 2073 \\
\hline
\end{tabular}

A

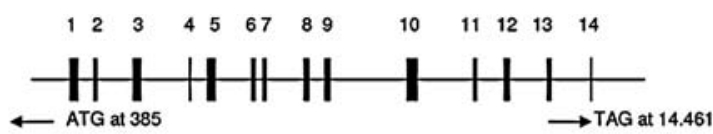

MW

(bp) $1 \begin{array}{lllllllllllllll}1 & 2 & 3 & 4 & 5 & 6 & 7 & 8 & 9 & 10 & 11 & 12 & 13 & 14\end{array}$

B
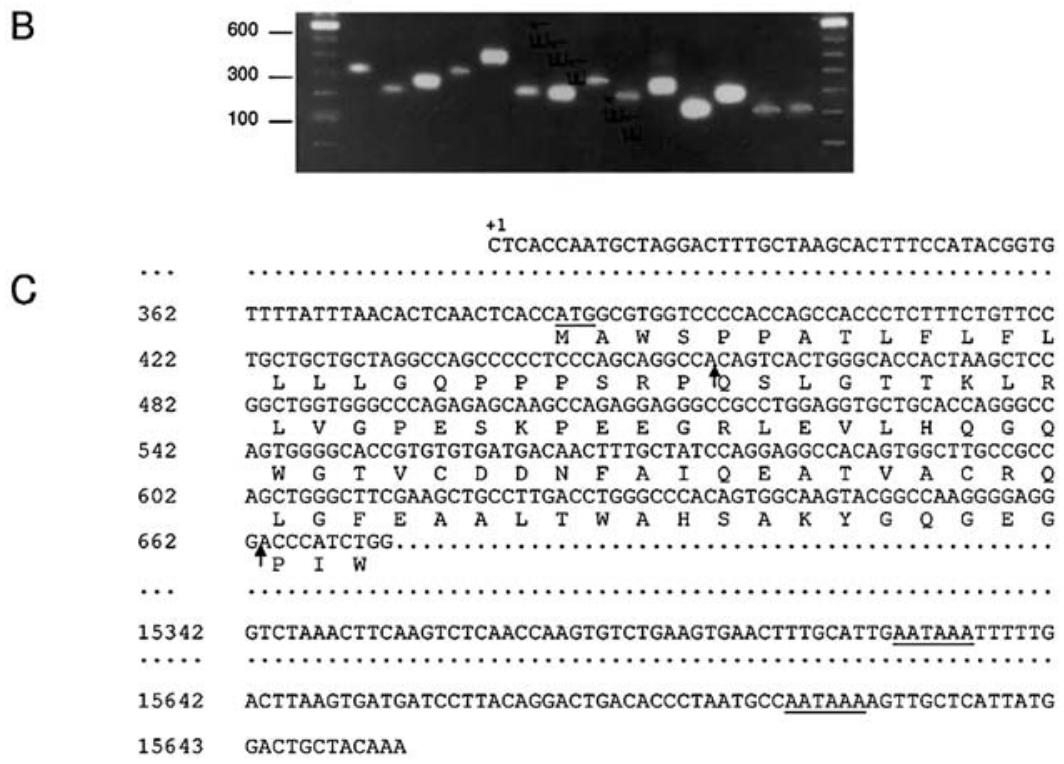

Figure 1. Structural features of the LOXL4 gene. (A) Schematic representation of exons and introns of the LOXL4 gene. Codon numbers for ATG and TAG are based on human BAC clone AL 139241 sequences. (B) Ethidium bromide agarose gel electrophoresis showing exons 1-14 (lanes 1-14), of the LOXL4 gene as fragments amplified by PCR using genomic DNA isolated from normal squamous epithelial cells. (C) Nucleotide and elucidated amino acid sequences of the first exon and a part of the 3'UTR sequence. The translation initiation codon ATG and the polyadenylation signals are underlined. The $\mathrm{Q}(\mathrm{Gln})$ to the right of the first vertical arrow is the amino terminus of the mature polypeptide and the arrow between $\mathrm{G}$ and $\mathrm{A}$ marks the 3 ' end of the first exon

PCR amplified using genomic DNA from both HNSCC and normal epithelial cells. Comparison of the amplified sequences derived from HNSCC cells (Fig. 3) with those derived from normal control epithelial cells and the corresponding region of the high throughput human genomic sequence (accession no. AL139241) detected no mutations or sequence differences. Sequence motif analysis for DNA binding proteins identified several binding sites for transcription factors and these are indicated in Fig 3. A TATAbox was found at -25 and the TRE sequence, TGACTCA (TPA-responsive element), a binding site for transcription factor AP-1, was noted at -75 . The GATA domain was 


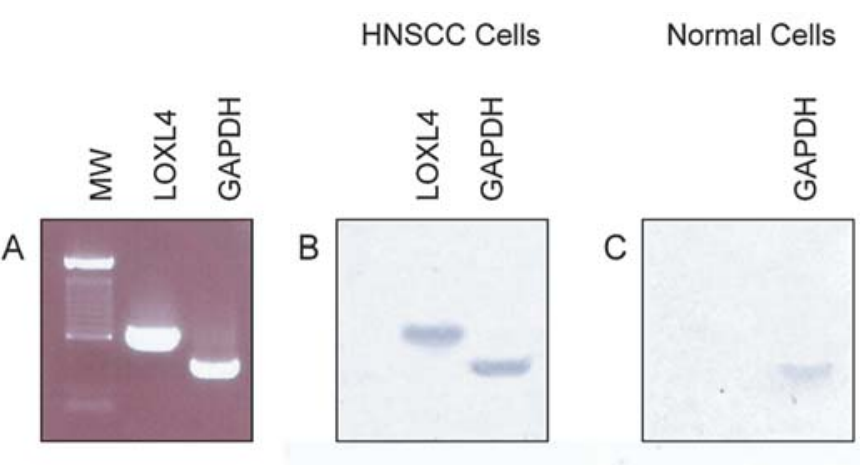

Figure 2. Nuclear run-on analysis of differential expression of LOXL4 in HNSCC and normal epithelial cells. After ethidiumbromide agarose gel electrophoresis of LOXL4 and G3PDH-cDNA (A) the fragments were transferred onto a positive-charged nylon membrane, immobilized by UVcrosslinking, and hybridized using $\left[\alpha^{32} \mathrm{P}\right] \mathrm{dUTP}$ labeled RNA which was synthesized in the nuclear run-on procedure. De novo transcription of the LOXL4 gene is detectable in carcinoma cells (B) in contrast to normal epithelial cells (C). The presence of the G3PDH gene fragment in both cell types demonstrates the reliability of the run-on transcription.

located at $-113,-506$, and -669 respectively, and the RFX1 transactivator-binding site, GGAA, at -149 . The motif GGCGGC at -181 is a consensus sequence identified as binding site for the transcription factor Sp1. CCAAT (CP1factor) at -257 and -892 and the repetitive sequence motifs GAAA at -310 and 329 , are known conserved recognition sequences for DNA binding proteins. No consensus sequences typical for a GC box were present.

Increased LOXL4 promoter activity in HNSCC cells. To confirm that the higher LOXL4 transcription rate in carcinoma cells correlates with the gene promoter activity, seven LOXL4/pGL3 enhancer constructs including SV-40 promoter-derived sequences were generated and analyzed by DLA. The differences in LOXL4 promoter construct activities were determined by normalizing data for transfection efficacy using firefly-luciferase activity data as internal control. The transient expression of the human LOXL4 gene promoter constructs increased the luciferase reporter activity for all constructs in carcinoma cells, but not in cells with normal epithelial phenotype. The results from the mean of nine separate experiments are illustrated in Fig. 4. The promoter constructs REP1-4 that contained nucleotides -180 to +1 and REP1- 6 that contained nucleotides -270 to +1 showed the highest, 7 - and 8 -fold, increase in luciferase activity in carcinoma cells above the levels of the same reporter gene activities we have measured in normal epithelial cells $(\mathrm{p}=0.01)$. Furthermore, both constructs demonstrated the highest overall activity in carcinoma cells, supporting the role of these promoter regions in LOXL4 gene overexpression, while promoter constructs with the remaining five binding sites appeared essential for basal promoter activity. There was a substantial difference between the two cell types in the absolute luciferase level of each individual REP ( $\mathrm{p}=0.02$ ), but the activity profiles were somewhat similar, suggesting that some of the basic regulatory mechanisms for LOXL4 transcription may not have been altered in the course of malignant transformation of epithelial cells in HNSCC.

Identification of potential binding sites for transcription factors. To determine whether differences in the amounts and/or affinities of DNA binding proteins are associated with the increase of LOXL4 expression in HNSCC cells, we have analyzed selected DNA binding proteins that may directly affect LOXL4 transcription. For EMSA nuclear extracts derived from HNSSC and normal cells were incubated with $\left[\gamma^{32} \mathrm{P}\right] \mathrm{dATP}-$ labeled oligonucleotides REP1-7 that correspond

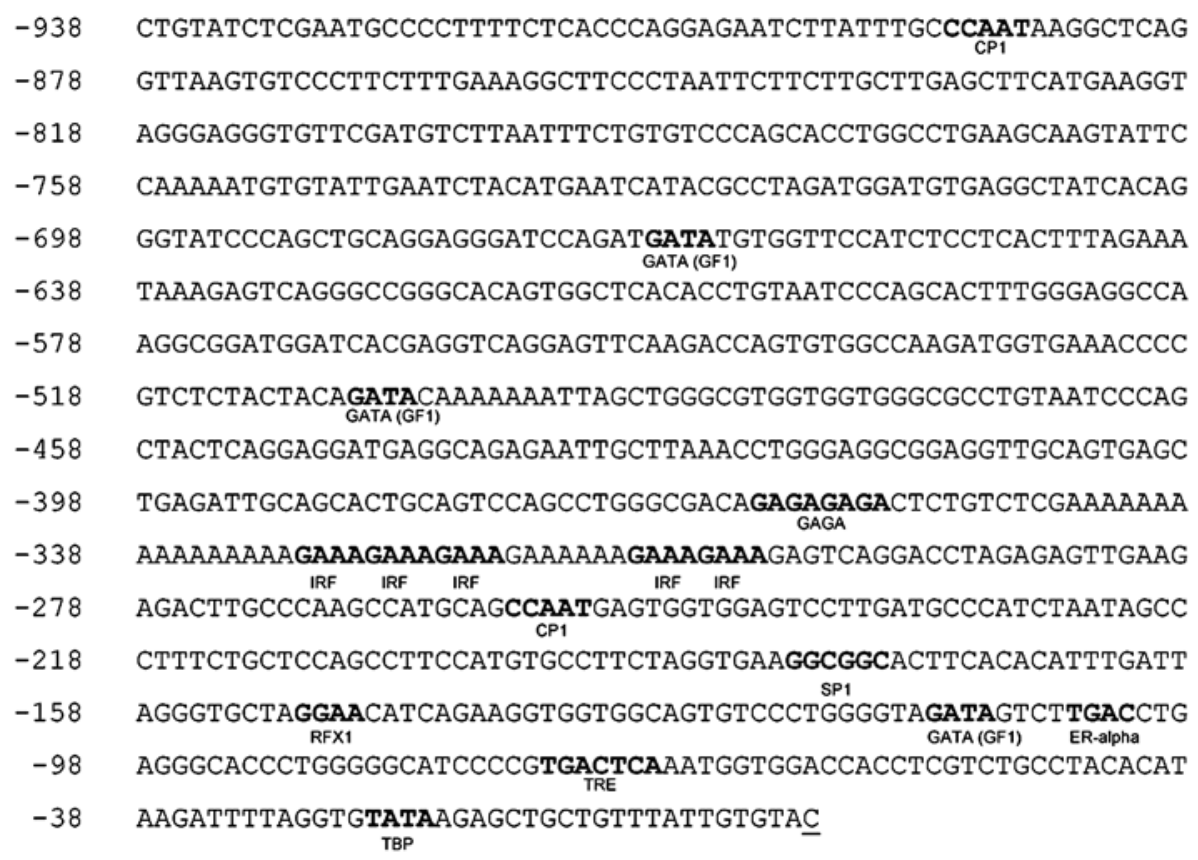

Figure 3. Nucleotide sequences of the 5 ' flanking domain of the LOXL4 gene. The underlined C designated as +1 is the start site of transcription. The sequence motifs in bold are consensus binding sites for transcription factors identified using the Matlnspector2 V2.2 program. 


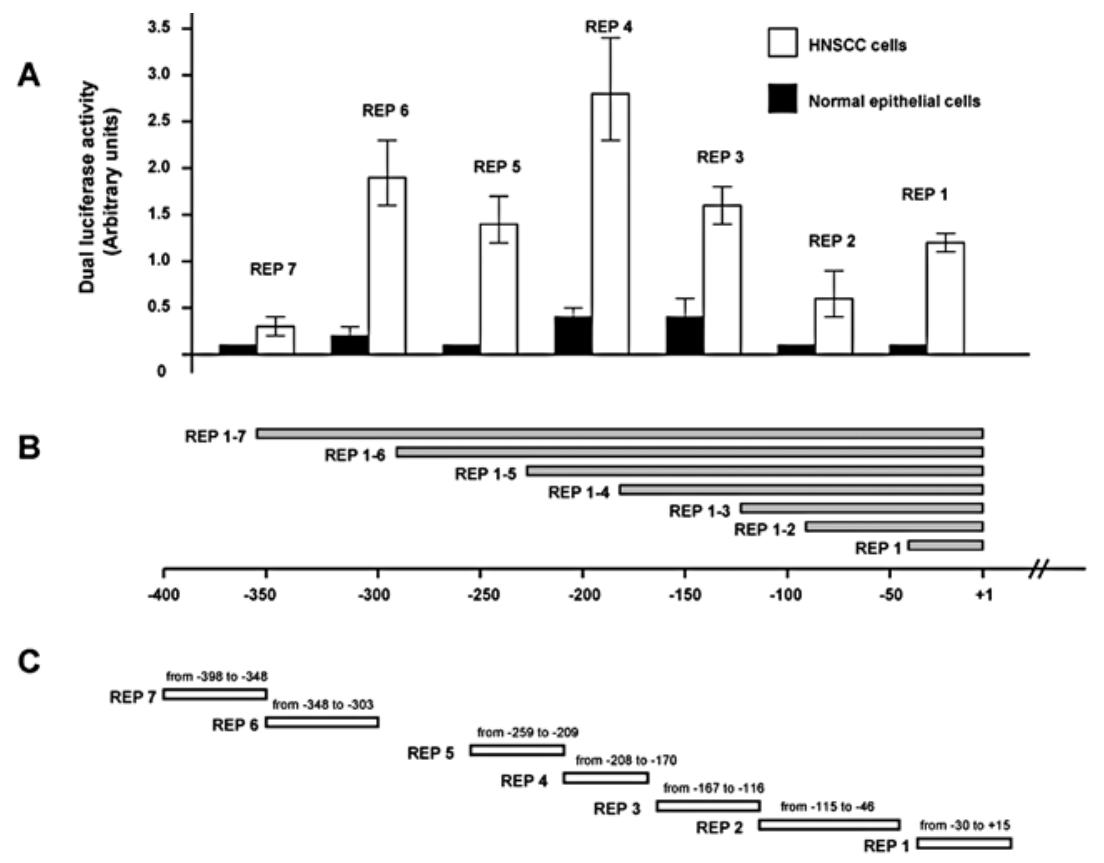

Figure 4. Functional analysis of the LOXL4 promoter. Transient transfections and analyses were performed using dual luciferase activity (DLA) assays in HNSCC cells in comparison with normal epithelial cells using plasmid constructs containing a series of promoter region deletion constructs of the LOXL4 gene. To monitor the transfection efficiency, each transfected cell culture was co-transfected with the pGL3 control vector the activity of which was designated as zero. (A) Columns represent the mean dual luciferase (DLA) values for nine independent experiments. The error bars show standard deviation of four single measurements per culture. Activity was calculated in $\mathrm{ng} / \mathrm{ml}$ of the calibration standard. Values show relative light units (RLU) of firefly luciferase in relation to RLU of renila luciferase (mean \pm standard deviation) normalized for transfection efficiency with firefly luciferase activity as an internal control. (B) pGL3 plasmid constructs containing LOXL4 gene regulatory regions. Each individual promoter fragment is numbered starting from the transcription initiation site +1 . (C) Oligonucleotides REP1-7 that correspond to most 5' parts of the DNA regions are shown under B. The oligonucleotide probes contain specific sequence motifs for transcription factor recognition and were designed for EMSA.

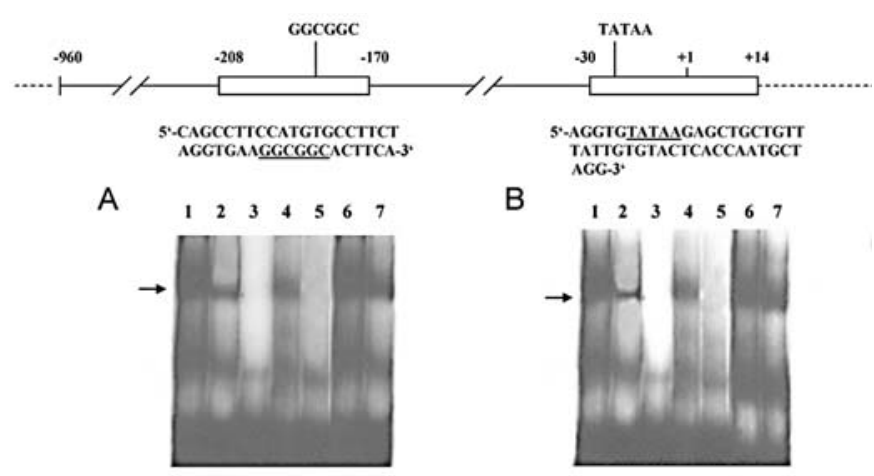

Figure 5. EMSA assays demonstrate nuclear protein binding reactions to LOXL4 promoter fragments using $\left[\gamma^{32} \mathrm{P}\right] \mathrm{dATP}-$ labeled oligonucleotides (REPs). (A) Binding reactions of REP4 containing the GGCGGC binding site for Sp-1. (B) Binding reactions of REP1 containing the TATA site for TBP. Numbers above the lanes indicate reactions with (1) nucleic extract of carcinoma cells, (2) nucleic extracts of normal epithelial cells, (3) negative control without protein, (4) sequence specific competitor for carcinoma cells and (5) for normal cells, (6) unspecific competitor sequences for carcinoma cells and (7) for normal cells. The arrows on the left of the panels indicate band shifts present in lanes 1 and 2 . In lanes 4 the band shifts remained unaffected in spite of competitor.

to sections of the promoter region from nucleotides -398 to +15 and contain specific DNA binding protein recognition sequences (Fig. 4C). In experiments performed for oligonucleotides REP2, REP3, and REP5 no significant band shifts were detected. However, significant band shifts were noted when REP1 and REP4 (Fig. 5) oligonucleotides were used in these assays. To further test if the observed shifted bands were specific for the transcription factors contained within these sequences, i.e. REP1 specific for TBP and REP4 specific for Sp1, competition tests with an excess of cold probes were performed. The cold probes eliminated the shifted bands in normal epithelial cells, but not in HNSCC cells suggesting that the band configuration, shown in Fig. 5A, was the result of specific Sp1-target DNA binding in HNSCC cells, whereas that in Fig. 5B resulted from specific TBPtarget DNA binding ( $\mathrm{p}=0.02$ ) in HNSCC cells. Further significant band shifts were detected for fragments REP6 and REP7. These results point to the sequence-specific binding of the transcription factors CP1 (within REP6), and IRF1 and IRF2 (within REP7) within the LOXL4 promoter, but for these sites similar binding parameters were noted for the two cell types (data not shown).

\section{Discussion}

Although the LOXL4 cDNA has been described previously $(1,2)$, there was no information regarding the genomic organization of the LOXL4 gene. We have identified 2,278 kb of LOXL4 gene coding sequences distributed in 14 exons within a human genomic clone along with flanking 5 ' and 3 ' untranslated sequences, and showed that the gene spans about $14 \mathrm{~kb}$ on chromosome 10q24.2. Our Northern blot analyses revealed a single LOXL4 mRNA of $3.9 \mathrm{~kb}$ in all HNSCC and normal cell samples (13). This value was in close agreement with the previously reported mRNA size of 
$4 \mathrm{~kb}$ in skeletal muscle, testis and pancreas (1) but somewhat different from the $3.5 \mathrm{~kb}$ band we have detected previously in other normal human tissue samples (2). Some of these mRNA size differences can be due to detection methods, but can also represent tissue and/or cell type-specific mRNA size differences. The presence and alternative epithelial cell specific use of the two polyadenylation signals we have identified within the 3' UTR region of the LOXL4 gene, may explain these differences in mRNA size. Northern hybridization experiments of HNSCC biopsies and cell lines consistently revealed one LOXL4 mRNA species, without evidence for the utilization of both or a second polyadenylation site in these cells. However, these polyadenylation sites might be used differentially in other tissue types and result in transcripts of different lengths that we have noted in normal tissues.

Of members of the LOX gene family, we have only analysed regulatory DNA sequence elements for LOX within the 5' flanking domain and found differentially increased CAT reporter activities for promoter elements, and in addition sequences within intron-1, in normal cell lines, and $\mathrm{cHa}$ ras-transformed osteosarcoma cells (16). These differences in CAT activity in the normal and ras-transformed cells suggested a complex interaction between cis-acting domains and trans-acting transcriptional factors in the $5^{\prime}$ promoter domain and the first exon of the LOX gene. The LOX promoter was also reported to be controlled by cross-talk between positive and negative cis-acting regions that are differentially active in various cell types $(17,18)$.

Analysis of the 5' flanking domain of the LOXL4 gene revealed that the sequence is $\mathrm{G}+\mathrm{C}$ rich up to about -180 , where it becomes relatively $\mathrm{A}+\mathrm{T}$ rich. We have structurally and functionally analyzed different parts of the 5 ' untranslated region using reporter and transfection systems. While there were no housekeeping gene-typical GC-box sequences present, GC-box-like sequences and multiple known transcription factor binding sites were identified. While all reporter constructs exhibited overall increased transcription in HNSCC cells, two constructs, REP-4 and REP-6 demonstrated considerably increased promoter activity in HNSCC cells compared to normal epithelial cells.

As consensus sequences, such as GC or CCAAT, found in promoters often lead to alteration of promoter activity due to their frequent mutation or deletion (19), we have tested HNSCC derived LOXL4 DNA sequences for mutations. Sequence analysis of the full promoter and coding regions of the LOXL4 gene revealed no genetic alteration between DNA sequences in HNSCC and those derived from normal epithelial cells and database sequences. In tumor cells in vivo DNA methylation/demethylation is known to be one of the differential gene regulatory mechanisms and we have considered this possibility, particularly as there were GC rich promoter regions present within the LOXL4 promoter. But in our experiments, increased promoter activity in HNSCC cells as a consequence of DNA methylation could be ruled out, as the promoter deletion constructs we have tested in transfected cells were generated by PCR under methylase-free condition.

To further determine the mechanistic basis for differential promoter activity between HNSCC and normal epithelial cells, we have evaluated different binding affinities for transcription factor binding sites within the LOXL4 promoter using specific oligonucleotide probes and nuclear extracts from HNSCC and normal control cells. The regulation of transcription is commonly based on the interactions between general and basal transcription factors and between general transcription factors with each other (20-22). To evaluate these interactions in HNSCC we have investigated the enhancing and/or silencing effect of several fragments of the LOXL4-promoter on the transcription rate in reporter gene studies and subsequent EMSA. Two promoter fragments, REP-1 specific for TBP and REP-4 specific for Sp1, showed differentially increased expression in HNSCC cells and the specific binding of these factors was further confirmed in competition experiments. TBP that recognizes the TATA box at -25 is responsible for the formation of preinitiation complex where DNA-dependent RNA polymerase II starts transcription. Normally, the binding of TBP to the DNA is very slow, because the formation of non-DNA-associated homo-dimers of TBP competes with its binding to the DNA. But if the concentration of TBP is increased compared to the normal level, the balance changes towards DNA-binding which then accelerates the initiation of transcription (23). Based on our results we hypothesized that the transcription of the LOXL4-gene is initiated more rapidly in tumor cells and the EMSA data supported this hypothesis for REP-1. The stronger band intensity we have observed using tumor cell extracts is an indicator of an increased concentration of TBP that can possibly lead to faster initiation of transcription in these cells. Recently, a similar mechanism has also been described for colon carcinoma cells (24).

The consensus 12-O-tetradecanoylphorbol-13-acetate (TPA)-responsive element (TRE) at -75 found in REP-2 is a target DNA control element of distinct signal transduction pathways and is very similar to CRE (TGACGTCA) which is necessary for cAMP induction in the phosphoenol-pyrovate carboxykinase gene (25). This similarity may provide a route for different intracellular signals to be integrated at the level of transcriptional control $(26,27)$. The sequence GGAA at -149 (REP-3) is a transcription factor binding motif for a large number of conserved transcription factors many of which have been implicated in tumor progression such as Ets with critical role in control of cellular changes during epithelial-mesenchymal transition and metastasis $(28,29)$.

The sequence GGCGGC at -181 , that in REP-4 demonstrated specific and differential EMSA, corresponds to sequences identified as binding sites for the transcription factor Sp1 (30). This sequence specific activator generally increases the rate of the transcription factor subunit IID (TFIID) association with the promoter and interacts directly with TBP-associated factor (TAF) components of TFIID (31). Another important transcription factor binding motif is a CCAAT at -257 (REP-5) that operates in interactions with multiple boxes and conserved motifs $(32,33)$, though we did not observe EMSA for this site in HNSCC or normal epithelial cells. The tandem repeated GAA sequences located between -329 and -306 were positive within REP-7 in EMSA are recognition sites for IRF (interferon regulatory factor) known to play an important role in oncogenesis (34). 
In summary, there are many heterogeneous sequence motifs within the regulatory region of the human LOXL4 gene, identified as transcription factor-binding motifs $(19,28,29,32-37)$. Some of these (GATA, GGAA, CCAAT, GAAA) may not participate in the regulation of the LOXL4 gene expression in promoting transcription factor interactions. Our experiments clearly revealed the significance of differential transcription activating factor binding for TBP within REP-1 and Sp1 within REP-4 in HNSCC cells suggesting that these factors may be the primary responsive sites for the regulation of LOXL4 overexpression in HNSCC.

\section{Acknowledgements}

We are grateful to Anne-Marie Roen (Department of Otorhinolaryngology, Head and Neck Surgery, University Kiel) for technical assistance. This work was supported in part by grants from the IZKF University of Kiel, Germany and NIH grant AR47662 to K.C.

\section{References}

1. Mäki JM, Tikkanen H and Kivirikko KI: Cloning and characterization of a fifth human lysyl oxidase isoenzyme: the third member of the lysyl oxidase-related subfamily with four scavenger receptor cysteine-rich domains. Matrix Biol 20: 493-496, 2001.

2. Asuncion L, Fogelgren, B, Fong KS, Fong SF, Kim Y and Csiszar K: A novel human lysyl oxidase-like gene (LOXL4) on chromosome 10q24 has an altered scavenger receptor cysteine rich domain. Matrix Biol 20: 487-491, 2001.

3. Kagan HM and Trackman PC: Properties and function of lysyl oxidase. Am J Respir Cell Mol Biol 3: 206-210, 1991.

4. Csiszar K: Lysyl oxidases: a novel multifunctional amine oxidase family. Prog Nucleic Acid Res Mol Biol 70: 1-32, 2001.

5. Jourdan-Le Saux C, Tronecker H, Bogic L, Bryant-Greenwood GD, Boyd CD and Csiszar K: The LOXL2 gene encodes a new lysyl oxidase-like protein and is expressed at high levels in reproductive tissues. J Biol Chem 274: 12939-12944, 1999.

6. Jourdan-Le Saux C, Tomsche A, Ujfalusi A, Jia L and Csiszar K: Central nervous system, uterus, heart and leukocyte expression of the LOXL3 gene, encoding a novel lysyl oxidase-like protein. Genomics 74: 211-218, 2001.

7. Hein S, Yamamoto SY, Okazaki K, Jourdan-LeSaux C, Csiszar K and Bryant-Greenwood GD: Lysyl oxidases: expression in the fetal membranes and placenta. Placenta 22: 49-57, 2001.

8. Csiszar K, Fong SF, Ujfalusi A, Krawetz SA, Salvati EP, Mackenzie JW and Boyd CD: Somatic mutations of the lysyl oxidase gene on chromosome 5q23.1 in colorectal tumors. Int J Cancer 97: 636-642, 2002.

9. Kirschmann DA, Seftor EA, Fong SFT, et al: A molecular role for lysyl oxidase in breast cancer invasion. Cancer Res 62: 4478-4483, 2002.

10. Payne SL, Fogelgren B, Hess AR, et al: Lysyl oxidase regulates breast cancer cell migration and adhesion through a hydrogen peroxide-mediated mechanism. Cancer Res 24: 11429-11436, 2005.

11. Erler JT, Bennewith KL, Nicolau M, et al: Lysyl oxidase is essential for hypoxia-induced metastasis. Nature 7088: 1222-1226, 2006.

12. Holtmeier C, Görögh T, Beier U, et al: Overexpression of a novel lysyl oxidase-like gene in human head and neck squamous cell carcinomas. Anticancer Res 23: 2585-2592, 2003.

13. Görögh T, Weise JB, Holtmeier C, et al: Selective upregulation and amplification of the lysyl oxidase like-4 (LOXL4) gene in head and neck squamous cell carcinoma. J Pathol 212: 74-82, 2007.

14. Weber J, Jelinek W and Darnell JE: The definition of a large viral transcription unit late in Ad2 infection of HeLa cells: mapping of nascent RNA molecules labeled in isolated nuclei. Cell 10: 611-616, 1997.

15. Bradford MM: A rapid and sensitive method for the quantitation of microgram quantities of protein utilizing the principle of protein-dye binding. Anal Biochem 72: 248-254, 1976.
16. Csiszar K, Entersz I, Trackman PC, Samid D and Boyd CG: Functional analysis of the promoter and first intron of the human lysyl oxidase gene. Mol Biol Rep 23: 97-108, 1996.

17. Jourdan-Le Saux C, Gleyzal C, Raccurt M and Sommer P: Functional analysis of the lysyl oxidase promoter in myofibroblast-like clones of 3T6 fibroblast. J Cell Biochem 64: 328-341, 1997.

18. Reynaud C, Gleyzal C and Jourdan-Le Saux C: Comparative functional study of the lysyl oxidase promoter in fibroblasts, ras-transformed fibroblasts, myofibroblasts and smooth muscle cells. Cell Mol Biol 45: 1237-1247, 1999.

19. Maity SN, Golumbek PT, Karsenty G and de Crombrugghe B: Selective activation of transcription by a novel CCAAT binding factor. Science 241: 582-585, 1998.

20. Tjian R and Maniatis T: Transcriptional activation: a complex puzzle with few easy pieces. Cell 77: 5-8, 1994.

21. Pugh BF: Mechanisms of transcription complex assembly. Curr Opin Cell Biol 8: 303-311, 1996.

22. Goodrich JA, Cutler G and Tjian R: Contacts in context promoter specificity and macromolecular interactions in transcription. Cell 84: 825-830, 1996.

23. Hoopes BC, LeBlanc JF and Hawley DK: Kinetic analysis of yeast TFIID-TATA box complex formation suggests a multistep pathway. J Biol Chem 267: 11539-11547, 1992.

24. Johnson EM, Kinoshita Y and Daniel DC: A new member of the MCM protein family encoded by the human MCM8 gene, located contrapodal to GCD10 at chromosome band 20p12.313. Nucl Acids Res 31: 2915-2925, 2003.

25. Short JM, Wynshaw-Boris A, Short HP and Hanson RW: Characterization of the phosphoenolpyruvate carboxykinase (GTP) promoter-regulatory region. II. Identification of cAMP and glucocorticoid regulatory domains. J Biol Chem 261: 9721-9726, 1986.

26. Hai $\mathrm{T}$ and Curran $\mathrm{T}$ : Cross-family dimerization of transcription factors Fos/Jun and ATF/CREB alters DNA binding specificity. Proc Natl Acad Sci USA 88: 3720-3724, 1991.

27. Masquilier D and Sassone-Corsi P: Transcriptional cross-talk: nuclear factors CREM and CREB bind to AP-1 sites and inhibit activation by Jun. J Biol Chem 267: 22460-22466, 1992.

28. Hsu T, Trojanowska M and Watson DK: Prospect Ets proteins in biological control and cancer. J Cell Biochem 91: 896-903, 2004.

29. Pastorcic M and Das HK: Alternative initiation of transcription of the human presenilin 1 gene in SH-SY5Y and SK-N-SH cells. The role of Ets factors in the regulation of presenilin 1. Eur $J$ Biochem 22: 4485-4494, 2004.

30. Dynan WS and Tjian R: Control of eukaryotic messenger RNA synthesis by sequence-specific DNA-binding proteins. Nature 316: 774-778, 1985 .

31. Goodrich JA and Tjian R: BP-TAF complexes selectivity factors for eukaryotic transcription. Curr Opin Cell Biol 6: 403-409, 1994.

32. Tasanen K, Oikarinen J, Kivirikko KI and Pihlajaniemi T: Promoter of the gene for the multifunctional protein disulfide isomerase polypeptide. Functional significance of the six CCAAT boxes and other promoter elements. J Biol Chem 267: 11513-11519, 1992

33. Maity SN, Sinha S, Ruteshouser EC and de Crombrugghe B: Three different Polypeptides are necessary for DNA binding of the mammalian heteromeric CCAAT binding factor. J Biol Chem 267: 16574-16580, 1992.

34. Fujii Y, Shimizu T, Kusumoto M, Kyogoku Y, Taniguchi T and Hakoshima T: Crystal structure of an IRF-DNA complex reveals novel DNA recognition and cooperative binding to a tandem repeat of core sequences. EMBO J 18: 5028-5041, 1999.

35. Oskouian B, Mendel J, Shocron E, Lee MA Jr, Fyrst H and Saba JD: Regulation of sphingosine-1-phosphate lyase gene expression by members of the GATA family of transcription factors. J Biol Chem 280: 18403-18410, 2005.

36. Edwards D, Murray JA and Smith AG: Multiple genes encoding the conserved CCAAT-box transcription factor complex are expressed in Arabidopsis. Plant Physiol 3: 1015-1022, 1998.

37. Lin R, Genin P, Mamane Y and Hiscott J: Selective DNA binding and association with the CREB binding protein coactivator contribute to differential activation of alpha/beta interferon genes by interferon regulatory factors 3 and 7. Mol Cell Biol 17: $6342-6353,2000$. 